\title{
Thermal properties of hot and dense matter: Influence of rapid rotation on protoneutron stars, hot neutron stars, and neutron star merger remnants
}

\author{
Polychronis Koliogiannis ${ }^{1, *}$ and Charalampos Moustakidis ${ }^{1}$ \\ ${ }^{1}$ Department of Theoretical Physics, Aristotle University of Thessaloniki, Greece
}

\begin{abstract}
The knowledge of the equation of state is a key ingredient for many dynamical phenomena that depend sensitively on the hot and dense nuclear matter, such as the formation of protoneutron stars and hot neutron stars. In order to accurately describe them, we construct equations of state at finite temperature and entropy per baryon for matter with varying proton fractions. This procedure is based on the momentum dependent interaction model and state-of-the-art microscopic data. In addition, we investigate the role of thermal and rotation effects on microscopic and macroscopic properties of neutron stars, including the mass and radius, the frequency, the Kerr parameter, the central baryon density, etc. The latter is also connected to the hot and rapidly rotating remnant after neutron star merger. The interplay between these quantities and data from late observations of neutron stars, both isolated and in matter of merging, could provide useful insight and robust constraints on the equation of state of nuclear matter.
\end{abstract}

\section{Introduction}

Neutron stars are born in the gravitational collapse of massive, degenerate stellar cores. Newly born stars are quite different than the ordinary ones, as they are hot and lepton rich [1-4]. In view of these, a study of hot neutron star matter is of increasing interest in relation to astrophysical phenomena, such as the neutron star mergers, the formation of protoneutron stars, and the formation of black holes. Their study requires the knowledge of the equation of state (EoS), which at several times the normal nuclear matter density remains unknown.

Due to the limit of the experimental data concerning the dense nuclear matter, constraints on the EoS are possible though the observation of non/slow rotating neutron stars. The most robust ones are related to the maximum mass of a neutron star, including the resent PSR J0740+6620 with $M=2.14_{-0.09}^{+0.10} M_{\odot}$ [5]. Nonetheless, the recent observations of binary neutron star mergers, GW170817 [6] and GW190425 [7], improved our knowledge of the EoS, especially in the intermediate region of densities. Furthermore, after the merger, the remnant and its maximum stable mass, spin period, and lifetime strongly depend on the dense matter properties at high temperatures and entropy. The latter is a valid candidate for constraints in the high density region.

In the present work we construct and apply in astrophysical processes, a set of thermodynamically consistent isothermal and isentropic EoSs, based on the parameterized cold one. Specifically, we dedicate a part for analyzing the free energy and the effect of the proton fraction related to dynamical processes. We explore the significant meaning of the constant baryon mass sequences and connect them with the microscopic properties of neutron stars. In

*e-mail: pkoliogi@physics.auth.gr addition, we focus on the extensive study on some of the bulk properties of both nonrotating and rotating at the massshedding limit hot neutron stars, including the moment of inertia, Kerr parameter, ratio of rotational kinetic to gravitational binding energy $T / W$, etc. Finally, we study the hot, rapidly rotating remnant of a binary neutron star merger in accordance with the derived EoSs.

The paper is structured as follows. In Section 2 we present the framework of the nuclear model. Section 3 provides the discussion of the results while in Section 4, the scientific remarks are pointed out. Finally, Appendix A includes the instability criteria.

\section{The Nuclear Model}

For the description of neutron stars, the EoSs have been constructed using the momentum-dependent interaction (MDI) model [8-10] and the data provided by Akmal et al. [11] for the APR-1 EoS (hereafter MDI+APR1). The schematic presentation of the cold, catalyzed matter of the MDI+APR1 EoS, for the pure neutron matter (PNM) and symmetric nuclear matter (SNM), is presented in Figure 1.

This parameterization is used for the construction of one cold EoS, 10 hot EoSs based on temperatures in the range $[1,60] \mathrm{MeV}$, and nine hot EoSs based on lepton fractions and entropies per baryon in the ranges $[0.2,0.4]$ and $[1,3] k_{B}$, respectively.

For the solid crust region of the cold case, we applied the EoS of Feynman et al. [12] and also Baym et al. [13], while for the finite temperature cases and the low-density region $\left(n_{b} \leq 0.08 \mathrm{fm}^{-3}\right)$, as well as the finite entropies per baryon and lepton fractions, the EoSs of Lattimer and Swesty [14] and the specific model corresponding to the incomprehensibility modulus at the saturation density of 


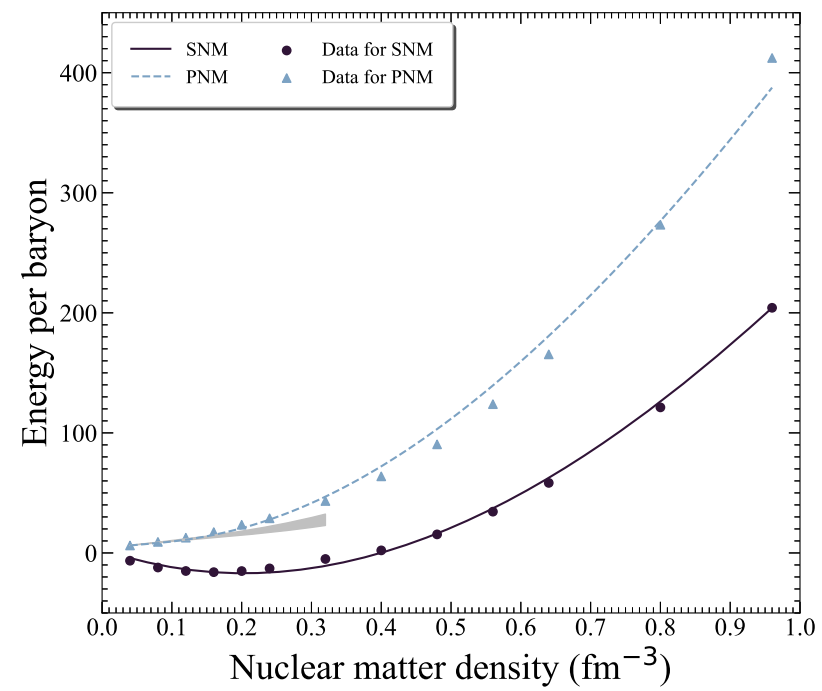

Figure 1. The fits of SNM and PNM of MDI+APR1 cold EoS. The SNM is presented by the circles and solid line, while the PNM is presented by the triangles and dashed line. The shaded region corresponds to benchmark calculations of the energy per particle of PNM extracted from Piarulli et al. [16].

SNM $K_{s}=220 \mathrm{MeV}$ are used (https://www.stellarcollapse. org).

For an extensive study and information about the construction of the EoSs see Ref. [15].

\section{Results and discussion}

\subsection{Free energy and proton fraction}

Figure 2 displays the free energy per particle as a function of the baryon density for (a) PNM and (b) SNM and temperatures in the range $[0,60] \mathrm{MeV}$. The free energy per particle is an important quantity directly related to the calculation of the proton fraction. The effect of the temperature in free energy is more pronounced at the low density region, while at the high density region, due to the quantum character of hadronic matter, the system tends to be more symmetric.

Figure 3 displays the proton fraction as a function of the baryon density for temperatures in the range $[0,60] \mathrm{MeV}$. As the proton fraction is related to the structure of neutron stars, similar sensitivity from temperature with the free energy is presented. More specific, while in the low density region there is a high dependence from temperature, in the high density region, the effects are very mild.

\subsection{Baryon mass sequences}

The baryon mass sequences are a way to study the evolution of a neutron star. In particular, we construct sequences related to the cooling of a neutron star, based on isothermal EoSs, and study the thermal evolution at the mass-shedding limit. In Figure 4 we display the Kepler frequency as a function of (a) the temperature and (b) the central baryon

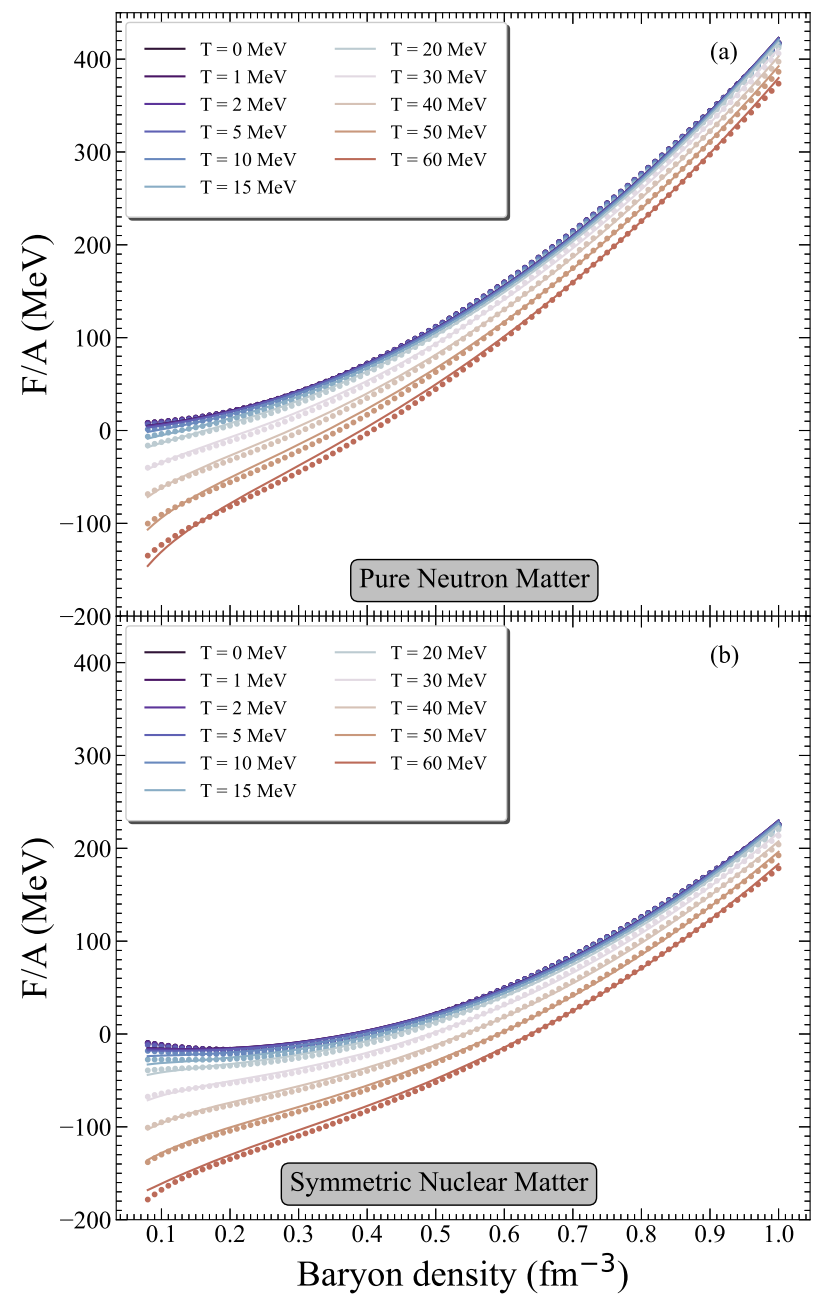

Figure 2. The free energy per particle as a function of the baryon density for (a) PNM and (b) SNM and temperatures in the range $[0,60] \mathrm{MeV}$. Fits are presented with the solid lines and data with circles (see also Ref. [15]).

density. Specifically, while Figure 4(a) presents the reduction of the Kepler frequency along the temperature, Figure 4(b) presents a twofold relation between the Kepler frequency and the central baryon density. For relatively low temperatures, $T<30 \mathrm{MeV}$, there is a linear dependence between the Kepler frequency and the central baryon density, assuming constant temperature in each case. In addition, for higher temperatures, a linear relation described as

$$
f\left(n_{b}^{c}\right)=-473.144+2057.271 n_{b}^{c} \quad(\mathrm{~Hz})
$$

holds on, independently of the selected baryon mass. This relation defines the allowed region for rotation with the Kepler frequency of a hot neutron star for a specific value of the central baryon density, and vice versa.

\subsection{Moment of inertia, Kerr parameter, and T/W ratio}

Moment of inertia is an important parameter that provides information about the structure of neutron stars. In Figure 5(a) we display the dimensionless moment of inertia as 


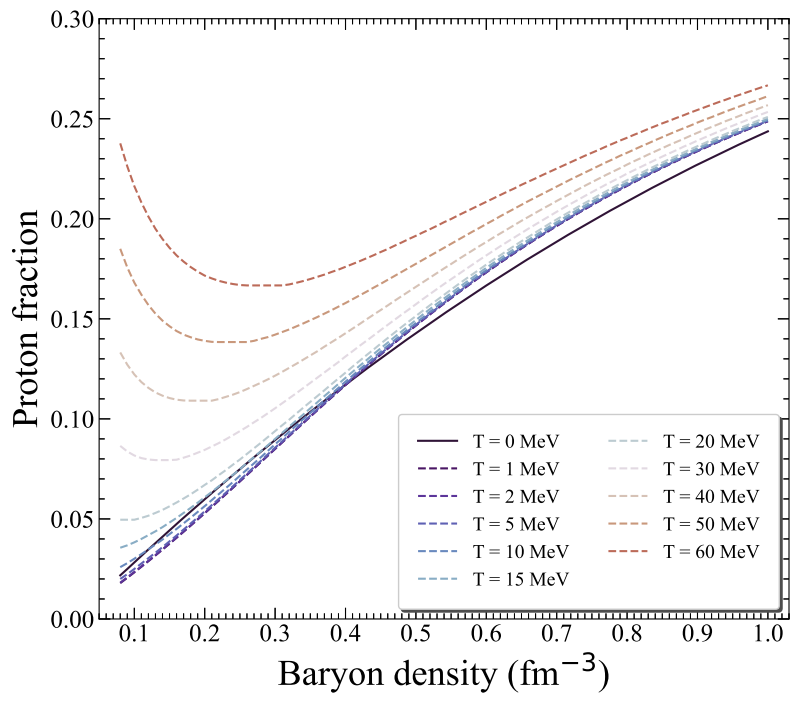

Figure 3. The proton fraction as a function of the baryon density for temperatures in the range $[0,60] \mathrm{MeV}$. The cold configuration is presented with the solid line and the hot ones with the dashed lines.

a function of the compactness parameter, defined as

$$
\beta=\frac{G}{c^{2}} \frac{M}{R}
$$

for isothermal neutron stars at the mass-shedding limit. The outcome is that in general, the increase of temperature leads to lesser compact stars with lower values of dimensionless moment of inertia than the cold neutron star.

Figure 5(b) displays the dimensionless moment of inertia as a function of the compactness parameter for isentropic neutron stars at the mass-shedding limit. In this case too, for a constant lepton fraction, the increase of the entropy per baryon leads to lesser compact stars with lower values of dimensionless moment of inertia than the cold neutron star.

Kerr parameter is a quantity directly related to neutron stars and black holes, defined as

$$
\mathcal{K} \equiv \frac{c}{G} \frac{J}{M^{2}} .
$$

We study the mass-shedding limit which is connected to the final fate of the collapse of a rotating compact star. Following the Refs. [17, 18], and the relations for angular velocity and moment of inertia, we found that the Kerr parameter, at the mass-shedding limit for a cold, catalyzed neutron star, is given by the simple universal expression

$$
\mathcal{K}_{\mathrm{k}} \simeq 1.34 \sqrt{\beta_{\max }}
$$

Assuming that, for the majority of realistic cold EoSs, the relation $0.24 \leq \beta_{\max } \leq 0.32$ holds, we concluded that $0.66 \leq \mathcal{K}_{\mathrm{k}} \leq 0.76[15]$.

Figures 5(c) and 5(d) display the Kerr parameter as a function of the gravitational mass for isothermal and isentropic neutron stars, respectively. As the temperature or the entropy per baryon increases, assuming a constant lepton
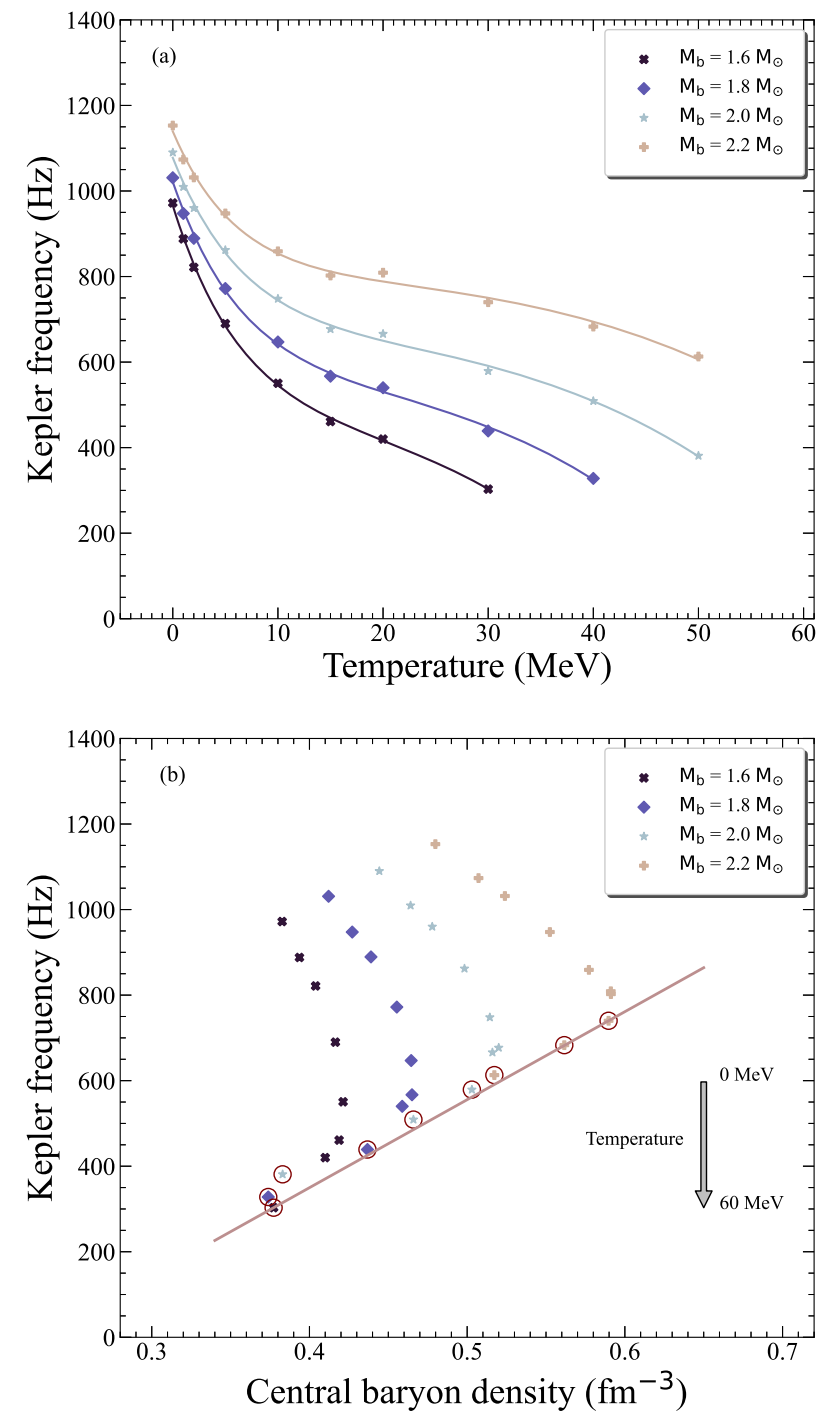

Figure 4. The Kepler frequency as a function of (a) the temperature and (b) the central baryon density for constant baryon mass sequences. (a) Solid lines correspond to fits (for more details see Ref. [15]). (b) The solid line corresponds to Equation (1) and open circles mark the high-temperature region $(T \geq 30 \mathrm{MeV})$.

fraction in the second case, the Kerr parameter decreases. The introduction of the temperature leads to the conclusion that the existed limit for Kerr black holes [19] and the one for neutron stars, through Equation (4), cannot be violated. Henceforth, the gravitational collapse of a hot, uniformly rotating neutron star, constrained to mass-energy and angular momentum conservation, cannot lead to a maximally rotating Kerr black hole.

Figures 5(e) and 5(f) display the angular velocity as a function of the ratio $T / W$ for isothermal and isentropic neutron stars, respectively. In neutron stars, the point that locates the nonaxisymmetric instability, which is responsible for the emission of gravitational waves, is defined via the ratio of rotational kinetic to gravitational binding energy $T / W$. In particular, instabilities driven by gravitational radiation for models with $M_{\mathrm{gr}}=1.4 M_{\odot}$ [20] would set in at $T / W \sim 0.08$. Figures 5(e) and 5(f) show that 

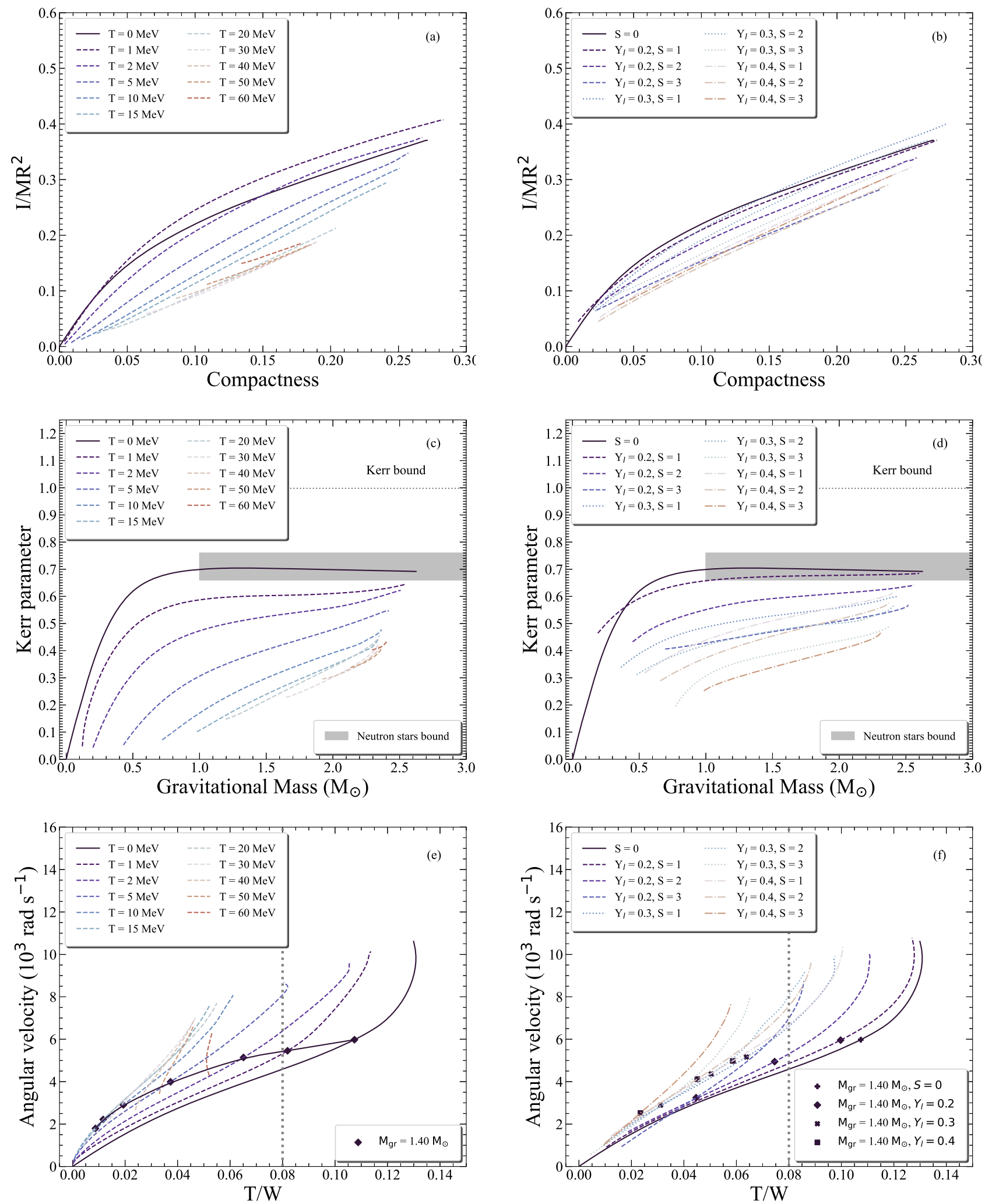

Figure 5. $(a, b)$ The dimensionless moment of inertia as a function of the compactness parameter for (a) isothermal and (b) isentropic neutron stars at the mass-shedding limit. (c,d) The Kerr parameter as a function of the gravitational mass for (c) isothermal and (d) isentropic neutron stars at the mass-shedding limit. The horizontal dotted line marks the Kerr bound for astrophysical Kerr black holes, $\mathcal{K}_{\text {B.H. }}=0.998$ [19]. The shaded region represents the limits for neutron stars from Equation (4). (e,f) The angular velocity as a function of the ratio of rotational kinetic to gravitational binding energy for (e) isothermal and (f) isentropic neutron stars at the mass-shedding limit. The vertical dotted line marks the critical value, $T / W=0.08$ [20], for gravitational radiation instabilities. Black plus sign, diamonds, squares, and crosses represent the $M_{\mathrm{gr}}=1.4 M_{\odot}$ configuration. For all Figures, the cold configuration is presented with the solid line while hot configurations, with the dashed ones. 
for sufficiently compact neutron stars, the nonaxisymmetric instability will set in before the mass-shedding limit is reached. The information that can be gained is that the maximum gravitational mass and the angular velocity will be lowered.

According to the analyzed quantities, useful insight can be gained for the hot, rapidly rotating remnant (at least $T \geq 30 \mathrm{MeV}$ for isothermal EoSs, $S=1$ and $Y_{l}=0.2$ for isentropic ones) after the neutron star merger, which is a compact object with neutron star matter. Assuming that the postmerger remnant is rotating at its mass-shedding limit, we study the maximum mass configuration. In this case, (a) the compactness parameter is constraint to $\beta_{\text {rem }}^{\text {iso }} \leq 0.19$ and $\beta_{\text {rem }}^{\text {ise }} \leq 0.27$, (b) the Kerr parameter is constraint to $\mathcal{K}_{\text {rem }}^{\text {iso }} \leq$ 0.42 and $\mathcal{K}_{\text {rem }}^{\text {ise }} \leq 0.68$, and (c) the ratio $T / W$ is constraint to $(T / W)_{\text {rem }}^{\text {iso }} \leq 0.05$ and $(T / W)_{\text {rem }}^{\text {ise }} \leq 0.127$ (the superscripts "iso" and "ise", correspond to isothermal and isentropic profiles). Two hypotheses can be made for the aftermath of a neutron star merger. In particular, in the isothermal case, it creates a lesser compact star than the cold neutron star with lower values of maximum gravitational mass and frequency, and stable toward dynamical instabilities, where for the isentropic aftermath, the object is comparable to the cold one [15].

\section{Remarks}

Thermal properties of hot and dense matter in the isolated neutron stars and in the matter of merging, remain an open problem. In order to gain insight into the role of thermal support, we constructed and applied in astrophysical phenomena a set of thermodynamically self-consistent EoSs (isothermal and isentropic). Specifically, we constructed nonrotating and uniformly rotating axisymmetric equilibrium sequences. Such equilibrium models may be acceptable for a first-order study of protoneutron stars, as well as of the postmerger remnant that has survived the initial highly dynamical and strongly nonaxisymmetric postmerger evolution and settled down into a stable quasi-equilibrium state [21].

The nuclear model which applied for the construction of the EoSs, presents merits compared to other models: (a) the thermal effects (both in isothermal and isentropic profiles) have been included in a self-consistent way, (b) the model is flexible enough to produce a wide range of EoSs by properly modifying the density dependence of the symmetry energy, (c) reproduces microscopic calculations concerning both the SNM and the PNM, (d) the momentum dependence of the potential interaction (which is absent in the majority of the proposed models) is in accordance with the terrestrial studies and experiments of heavy-ion reactions for both low and high densities and temperatures, and (e) the model ensures the causal behavior of the EoS at high densities.

For the numerical integration of the equilibrium equations we used the publicly available numerical code nrotstar from the $\mathrm{C}++$ Lorene/Nrotstar library [22] (for more details, see Ref. [15]).

In the postmerger phase, the baryon mass of the merger remnant is approximately conserved, an evidence that causes its dependence on temperature significant. Specifically, as the temperature rises, neutron stars lead to lower baryon masses. As in rapidly rotating configuration supramassive neutron stars exist, we study the mass-shedding limit in connection to the remnant. In particular, in the cold case, the baryon mass is $3.085 M_{\odot}$, while for a hot one at $T=30 \mathrm{MeV}$ is $2.427 M_{\odot}$ and one at $S=1$ is $3.05 M_{\odot}$. By assuming equal masses of merger components, these limits correspond to merger components of $\sim 1.5425, \sim 1.2135$, and $\sim 1.525 M_{\odot}$ baryon masses, respectively. Additionally, the immediate aftermaths of GW170817 [6] and GW190425 [7] had created hot, rapidly rotating remnants. Although the merger remnants are born with differential rotation, the uniform rotation approach can provide us with useful insight about the EoS. In the case of GW170817, a remnant with a total mass of $\sim 2.7 M_{\odot}$ has been created. In correlation with the MDI+APR1 EoS, with respect to baryon mass, only cold and isentropic neutron stars with uniform rotation can support the remnant. As for the GW190425 event, the existence of the $\sim 3.7 M_{\odot}$ remnant cannot be described only by uniform rotation.

Moment of inertia is a quantity that informs us about the distribution of matter in the star as it continuously changes its angular velocity and loses angular momentum due to radiation. The introduction of thermal effects in neutron stars lead to the conclusion that moment of inertia has lower values than the cold neutron star. This particular behavior is influenced by the unique interplay between the gravitational mass and the equatorial radius.

In our study, the maximum Kerr parameter existed in the cold neutron star. In particular, thermal support lead to lower values of the Kerr parameter. The outcome is that thermal support cannot lead a star to collapse into a maximally rotating Kerr black hole. In addition, it is observed that after $\sim 1 M_{\odot}$, while in the cold configuration the Kerr parameter is approximately constant, in hot configurations the Kerr parameter is an increasing function of the gravitational mass, leading to a unique maximum value.

The gravitational collapse to a black hole and the existence of stable supramassive neutron stars is related to the ratio $T / W$. In the specific case of the gravitational collapse due to gravitational radiation, the critical value of this ratio is $\sim 0.08$ for the $M_{\mathrm{gr}}=1.4 M_{\odot}$ configuration [20]. As thermal support leads to lower values for the ratio $T / W$, instabilities occurred by gravitational radiation never occur in a postmerger remnant or a hot, rapidly rotating neutron star. In some cases for low values of temperature or entropy per baryon, the ratio exceeds the limit. In this case, the critical value of $T / W$ set the limit for the maximum gravitational mass and angular velocity.

The effects of temperature on the EoS can be efficiently manifest though the evolutionary sequences of constant baryon mass. In particular, the attention is focused on the dependence of the Kepler frequency on the central baryon density. For temperatures $T \geq 30 \mathrm{MeV}$, the existence of a linear relation between these quantities, defines the allowed region of the pair of the central baryon density and corresponding Kepler frequency for a rotating hot neutron star at its mass-shedding limit. 
Future work should be focused on differential rotation, with emphasis on the various differential laws, and consider rotating configurations constructed with a broader set of hot microphysical EoSs. Finally, the postmerger remnant and its dynamical processes should be thoroughly investigated, as the LIGO and Virgo collaboration will provide us with more events of neutron star mergers.

The authors thank Prof. K. Kokkotas for his constructive comments on the preparation of the manuscript and Prof. L. Rezzolla for his useful and helpful considerations and clarifications.

\section{A Instabilities in Neutron Stars}

\section{A.1 Cold Neutron Stars}

The stability of cold rotating neutron stars is acquired via the turning-point criterion. It has to be noted that it is only a sufficient and not a necessary one. To be more precise, the neutral stability line is positioned to the left of the turningpoint line in $\left(M, \rho_{c}\right)$ space. Hence, the latter indicates that the star will collapse before reaching the turning-point line $[15,23,24]$.

\section{A.2 Hot Neutron Stars}

The stability of hot neutron stars is achieved with a specific version of the secular instability criterion of Friedman et al. [25], which follows the Theorem I of Sorkin [26]. We choose a continuous sequence of equilibria to be at a fixed baryon number $N_{\text {bar }}$ and total entropy of the neutron star $S_{\mathrm{t}}^{\mathrm{ns}}$, and the extremal point of the stability loss is [2]

$$
\left.\frac{\partial J}{\partial n_{b}^{c}}\right|_{N_{\mathrm{bar},}, S_{\mathrm{t}}^{\mathrm{ns}}}=0,
$$

where $J$ and $n_{b}^{c}$ are the angular momentum and central baryon density of the star, respectively.

In addition, a turning point in the sequence occurs where three out of four derivatives, $\partial M_{\mathrm{gr}} / \partial n_{b}^{c}, \partial M_{\mathrm{b}} / \partial n_{b}^{c}$, $\partial J / \partial n_{b}^{c}$, and $\partial S_{\mathrm{t}}^{\mathrm{ns}} / \partial n_{b}^{c}$, where $M_{\mathrm{gr}}$ and $M_{\mathrm{b}}$ denote the gravitational and baryon mass, vanish [15, 21, 27]. At this point, the turning-point theorem shows that the fourth derivative also vanishes, and the sequence has transitioned from stable to unstable.

\section{References}

[1] M. Prakash, I. Bombaci, M. Prakash, P.J. Ellis, J.M. Lattimer, R. Knorren, Phys. Rep. 280, 1 (1997)

[2] J.O. Goussard, P. Haensel, J.L. Zdunik, Astron. Astrophys. 321, 822 (1997)
[3] N. Glendenning, Compact Stars: Nuclear Physics, Particle Physics, and General Relativity (Springer, Berlin, 2000)

[4] F. Weber, Pulsars as astrophysical laboratories for nuclear and particle physics (UK: Institute of Physics, Bristol, 1996)

[5] H. Cromartie, E. Fonseca, S. Ransom, P.B. Demorest, Z. Arzoumanian et al., Nat. Astron. 4, 72 (2020)

[6] B.P. Abbott, R. Abbott, T.D. Abbott et al., Phys. Rev. Lett. 119, 161101 (2017)

[7] B.P. Abbott, R. Abbott, T.D. Abbott et al., Astrophys. J. 892, L3 (2020)

[8] C.C. Moustakidis, Phys. Rev. C 78, 054323 (2008)

[9] C.C. Moustakidis, Inter. J. Mod. Phys. D 18, 1205 (2009)

[10] C.C. Moustakidis, C.P. Panos, Phys. Rev. C 79, 045806 (2009)

[11] A. Akmal, V.R. Pandharipande, D.G. Ravenhall, Phys. Rev. C 58, 1804 (1998)

[12] R.P. Feynman, N. Metropolis, E. Teller, Phys. Rev. 75, 1561 (1949)

[13] G. Baym, C. Pethick, P. Sutherland, Astrophys. J. 170, 299 (1971)

[14] J.M. Lattimer, F.D. Swesty, Nucl. Phys. A 535, 331 (1991)

[15] P.S. Koliogiannis, C.C. Moustakidis, Astrophys. J. 912, 69 (2021)

[16] M. Piarulli, I. Bombaci, D. Logoteta, A. Lovato, R.B. Wiringa, Phys. Rev. C 101, 045801 (2020)

[17] P.S. Koliogiannis, C.C. Moustakidis, Phys. Rev. C 101, 015805 (2020)

[18] D.S. Shao, S.P. Tang, X. Sheng, J.L. Jiang, Y.Z. Wang, Z.P. Jin, Y.Z. Fan, D.M. Wei, Phys. Rev. D 101, 063029 (2020)

[19] K.S. Thorne, Astrophys. J. 191, 507 (1974)

[20] S.M. Morsink, N. Stergioulas, S.R. Blattnig, Astrophys. J. 510, 854 (1999)

[21] J.D. Kaplan, C.D. Ott, E.P. O’Connor, K. Kiuchi, L. Roberts, M. Duez, Astrophys. J. 790, 19 (2014)

[22] LORENE, Lorene: Langage objet pour la relativité numérique (1998), http: //lorene.obspm.fr/

[23] K. Takami, L. Rezzolla, S. Yoshida, Mon. Not. Roy. Astron. Soc. Lett. 416, L1 (2011)

[24] L.R. Weih, E.R. Most, L. Rezzolla, Mon. Not. Roy. Astron. Soc. Lett. 473, L126 (2017)

[25] J.L. Friedman, J.R. Ipser, R.D. Sorkin, Astrophys. J. 325, 722 (1988)

[26] R.D. Sorkin, Astrophys. J. 257, 847 (1982)

[27] M. Marques, M. Oertel, M. Hempel, J. Novak, Phys. Rev. C 96, 045806 (2017) 